\title{
NUMERICAL INVESTIGATION OF THE NEAR WAKE OF GENERIC SPACE LAUNCHER SYSTEMS AT TRANSONIC AND SUPERSONIC FLOWS
}

\section{Statnikov, C. Glatzer, J.-H. Meiß, M. Meinke, and W. Schröder}

Institute of Aerodynamics Aachen

RWTH Aachen University 5a Wüllnerstrasse, Aachen 52062, Germany

\begin{abstract}
Numerical simulations of the near wake of generic rocket configurations are performed at transonic and supersonic freestream conditions to improve the understanding of the highly intricate near wake structures. The Reynolds number in both flow regimes is $10^{6}$ based on the main body diameter, i. e., specific freestream conditions of ESA's Ariane launcher trajectory. The geometry matches models used in experiments in the framework of the German Transregional Collaborative Research Center TRR40. Both axisymmetric wind tunnel models possess cylindrical sting supports, representing a nozzle to allow investigations of a less disturbed wake flow. A zonal approach consisting of a Reynolds averaged Navier-Stokes (RANS) and a large-eddy simulation (LES) is applied. It is shown that the highly unsteady transonic wake flow at $\mathrm{Ma}_{\infty}=0.7$ is characterized by the expanding separated shear layer, while the Mach 6.0 wake is defined by a shock, expansion waves, and a recompression region. In both cases, an instantaneous view on the base characteristics reveals complex azimuthal flow structures even for axisymmetric geometries. The flow regimes are discussed by comparing the aerodynamic characteristics, such as the size of the recirculation region and the turbulent kinetic energy.
\end{abstract}

\section{INTRODUCTION}

The development of future launcher systems is among other issues constrained due to highly intricate and barely understood base flow physics. The dominating aerodynamic characteristics can lead to low-frequency pressure fluctuations in the wake, which are predominantly induced by fluctuations of a separated shear layer. Rollstin [1] determines the base drag to be up to $35 \%$ of the overall drag 
for supersonic freestream conditions, which could be even higher in the case of blunt rockets. The fluctuations may also exceed critical amplitudes and can lead to structural failure. During the transonic launch phase, in particular, the nozzle is ovalized due to the well-known buffeting problem. Therefore, the flow around the geometry is compared for Mach numbers of $\mathrm{Ma}_{\infty}=0.7$ and 6.0, corresponding to selected stages of a real rocket launch. In spite of simplified base geometries, the flow field remains highly complex, covering the interaction of the recirculation region with the separated shear layer, expansion waves and a recompression region. Since it is impossible to measure all the important flow features simultaneously, there is a basic demand for numerical simulations. The present paper shows the results of a new zonal approach for high Reynolds number numerical investigations and demonstrates the flow behavior in different flow regimes with an axisymmetric sting support at the base shoulder.

Reynolds averaged Navier-Stokes models are not capable to predict unsteady data and also fail to provide accurate results concerning the low pressure recirculation area at the base, while the predictions of the attached flow around the main body are quite satisfactory. Direct numerical simulations (DNS) are restricted to small Reynolds numbers and a small integration domain. Therefore, a zonal RANS/LES approach is applied. RANS simulations are used to predict the attached main body flow field while LES computations are applied to the unsteady wake flow using the RANS results as inflow conditions. The averaged turbulent viscosity of the RANS model is used to generate physical turbulent fluctuations at the inlet of the LES domain.

The article is organized as follows. First, a description of the computational technique and the meshes is presented in section 2. Subsequently, in section 3 the results of the numerical investigation are discussed, including a description of the general base flow topologies and a detailed description of the wake flow in the transonic and supersonic flow regime. Finally, some conclusions in section 4 summarize the article.

\section{NUMERICAL METHOD AND GEOMETRY SETUP}

\subsection{Zonal Approach}

The compressible Naver-Stokes equations are solved in a conservative form using a mixed central-upwind AUSM (advective upstream splitting method) scheme of a second-order accuracy with a low dissipation [2]. Splitting the inviscid fluxes into a convective and a pressure part and inserting the local speed of sound $c$ the convective part is reformulated using a Mach number weighted interpolation

$$
F^{c}=\frac{1}{2}\left[\left(\mathrm{Ma}_{+}+\mathrm{Ma}_{-}\right)\left(f_{-}^{c}+f_{+}^{c}\right)+\left|\mathrm{Ma}_{+}+\mathrm{Ma}_{-}\right|\left(f_{-}^{c}-f_{+}^{c}\right)\right] .
$$




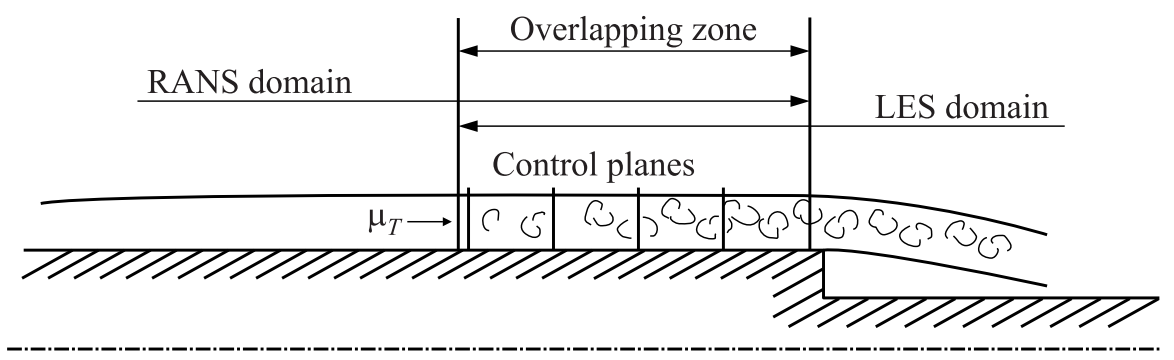

Figure 1 The RANS/LES overlapping zone with control panels

The fluxes $f_{ \pm}^{c}$ and the Mach numbers $\mathrm{Ma}_{ \pm}$at the cell boundaries are determined by left and right interpolated variables obtained using the monotonic upwind scheme for conservation laws (MUSCL) approach according to van Leer [3]. The non-Euler terms are discretized by a central scheme of a second-order accuracy. The temporal integration is performed by an explicit 5-stage Runge-Kutta scheme of a second-order accuracy, optimized for the maximum stability of a central scheme. For the calculation of the base area an LES is performed using the monotone integrated LES (MILES) approach [4]. That is, no explicit subgrid scale (SGS) model is implemented, but the dissipation of the numerical scheme serves as an implicit SGS model [5].

The flow around the main body is simulated using the one-equation RANS model according to Spalart and Allmaras [6]. The results of the RANS solution close to the trailing edge of the main body are used as inflow conditions for the base flow domain. The turbulent viscosity of the RANS model is used to generate physical turbulent fluctuations at the inlet of the LES domain [7-9]. The Reynolds stress is reconstructed starting from a normalized stochastic velocity signal, which is disturbed by a superposition of turbulent structures at prescribed geometric shape and a random sign and position. The body force $f$ is added to the wall-normal momentum equation at a number of control planes at different streamwise positions to match the turbulent flow properties of the LES with the given RANS values [10] as shown in Fig. 1. The additional forcing terms at the $i$ th position $x=x_{p, i}$ reads:

$$
f\left(x_{p, i}, r, \phi, t\right)=r(r, t)\left[u\left(x_{p, i}, r, \phi, t\right)-\langle u\rangle^{\phi, t}\left(x_{p, i}, r, t\right)\right]
$$

where $u$ is the velocity in the wall-normal direction; and $x, r$, and $\phi$ are the streamwise, wall-normal, and spanwise directions, respectively. The operator $\langle\cdot\rangle$ denotes arithmetic averaging in space and time according to

$$
\langle u\rangle^{\phi, t}\left(x_{p, i}, r, t\right)=\frac{1}{2 \pi \Delta T} \int_{t-\Delta T}^{t} \int_{0}^{2 \pi} u\left(x_{p, i}, r, \phi, t^{\prime}\right) d \phi d t^{\prime}
$$


at the period $\Delta T \approx 150 \delta / u_{\infty}$. The amplitude of the force term in Eq. (1) is controlled by a proportional plus integral controller

$$
r(r, t)=\alpha e(r, t)+\beta \int_{0}^{t} e\left(r, t^{\prime}\right) d t^{\prime}
$$

with the problem specific constants $\alpha, \beta$, and the error function $e$, which determines the difference between the target and the current profile of the Reynolds shear stresses

$$
e(r, t)=\left\langle u^{\prime} v^{\prime}\right\rangle^{*}\left(x_{p, i}, r\right)-\left\langle u^{\prime} v^{\prime}\right\rangle^{\phi, t}\left(x_{p, i}, r, t\right) .
$$

To keep the transition zone between the RANS and LES domains small, synthetic turbulence is added at the LES inflow according to Jarrin et al. [11]. In this method, turbulent structures are generated as a superposition of coherent vortices at the inflow boundary via a form function that meets specific spatial and temporal characteristics. Having $N$ synthetic vortices, the velocity signal at position $x$ is expressed as

$$
u_{i}^{\prime}(x)=\frac{1}{\sqrt{N}} \sum_{j=1}^{N} \epsilon_{j} f_{\sigma}\left(x-x_{j}\right)
$$

with $f_{\sigma}(x)$ being the form function of the vortex center, which is defined by a Gauss function implicitly meeting the two-point autocorrelation, and $\epsilon_{j}$ being a random number within the interval $[-1,+1]$. The final velocity at the inlet is given by

$$
u_{i}(x)=\bar{u}_{i}+a_{i, j} u_{i}^{\prime}
$$

in which the Cholesky transformation $a_{i, j}$ is applied to the prime velocity $u_{i}^{\prime}$. Due to the generation of the turbulent structures, the transition zone can be reduced to the length of approximately $3 \delta$. The zonal RANS/LES method was already successfully applied in $[9,12,13]$.

\subsection{Grid Topology and Boundary Conditions}

The simplified launcher model shown in Fig. 2 consists of a rounded conical top with an apex angle of $36^{\circ}$ and a cylindrical main body at the length to diameter ratio of 3 . The nose radius is 0.09 referred to the main body diameter. The configuration features a cylindrical support of 0.4 diameter mounted at the base of the main body. This support sting mimics the contour of the nozzle. For both the transonic and the supersonic configuration, two structured multiblock grids are used to resolve the flow field over the geometries. The first domain covers 


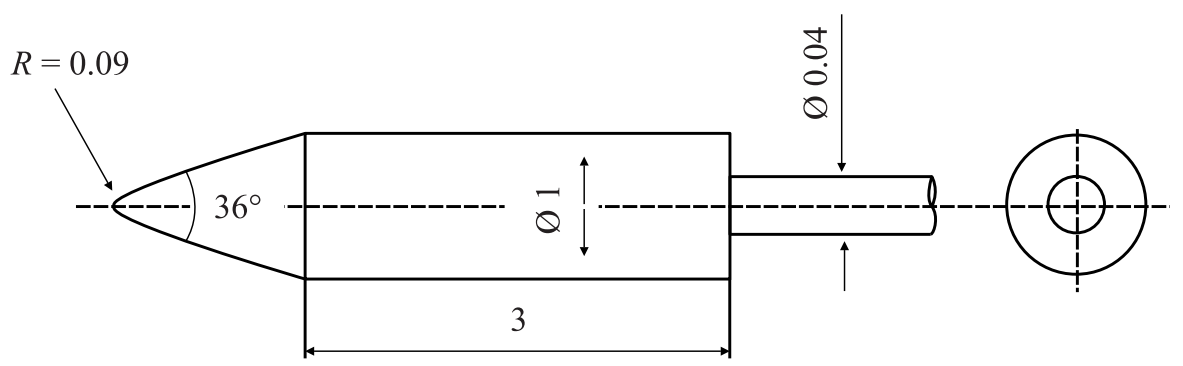

Figure 2 Geometry of the rocket configuration with the diameter as reference length

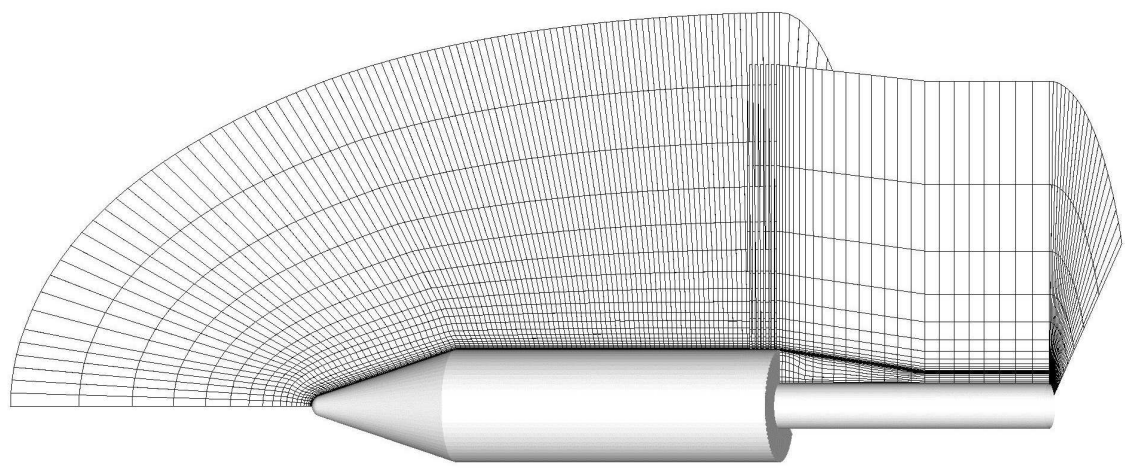

Figure 3 Grid topology for the transonic regime

the main rocket geometry, in which the fully three-dimensional (3D) RANS simulation is performed, and the second mesh encompasses the sting support and, thus, the highly time-dependent base area and the wake. In the second mesh, 3D LES computations are applied.

Figure 3 shows the two sections of the mesh for the transonic regime. The RANS section covering the main body at its whole circumference extends to physical values of approximately $10 D$ upstream of the conical top and in the radial direction, and its downstream boundary is located at the base shoulder. The grid is refined in the boundary layer and possesses a maximum resolution of $\left(\Delta x_{\text {wall }}^{+}, \Delta r_{\text {wall }}^{+}, \Delta \Phi_{\text {wall }}^{+}\right)=(500,1,150)$ at the model's surface, with $x$ being the streamwise, $r$ the wall-normal, and $\Phi$ the spanwise coordinate.

The LES domain for the transonic case comprises a sector of $60^{\circ}$ to save computational time. The grid extends to physical values from $-0.5 D$ to $2.5 D$ in the streamwise direction, with the base shoulder being the point of origin. This cre- 


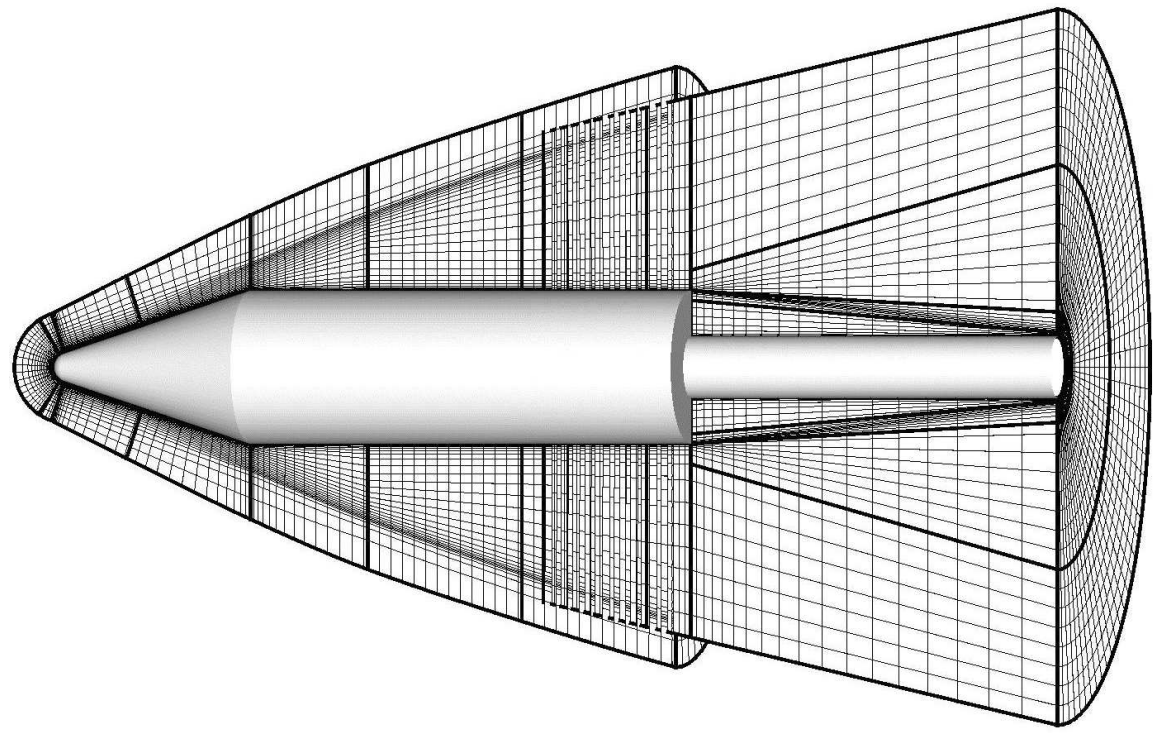

Figure 4 Grid topology for the supersonic regime

ates an overlapping zone of 0.5 diameter upstream from the base shoulder. The maximum resolution at the wall for the LES domain is $\left(\Delta x_{\text {wall }}^{+}, \Delta r_{\text {wall }}^{+}, \Delta \Phi_{\text {wall }}^{+}\right)$ $=(50,1,40)$. Additional refinement is realized along the separating boundary layer and the free shear layer. The total number of mesh points for the 60 degree part is $60 \cdot 10^{6}$.

The RANS and LES sections of the supersonic mesh are given in Fig. 4. The RANS section again covers the whole main body at its whole circumference and is generated so that it fully contains the oblique bow shock. The grid is refined in the boundary layer and along the bow shock and again possesses a maximum resolution of $\left(\Delta x_{\text {wall }}^{+}, \Delta r_{\text {wall }}^{+}, \Delta \Phi_{\text {wall }}^{+}\right)=(500,1,150)$ at the model's surface.

The supersonic LES domain extends to physical values of $-1.0 D$ and $2.5 D$ in the streamwise direction related to the base shoulder. As opposed to the transonic case, the mesh covers the full $360^{\circ}$ and contains the oblique shock in the radial direction. Consequently, the zonal overlapping zone spans up to 1 diameter upstream from the base shoulder. Again, the maximum resolution at the wall for the LES domain is $\left(\Delta x_{\text {wall }}^{+}, \Delta r_{\text {wall }}^{+}, \Delta \Phi_{\text {wall }}^{+}\right)=(50,1,40)$ and additional refinement in the base is realized along the separating boundary layer and the free shear layer. The total number of mesh points for the full partition is $16.2 \cdot 10^{6}$, and they are equally distributed over 16 blocks to allow load-balancing for an efficient parallel computation.

The boundary conditions are prescribed as follows. At subsonic boundaries, all variables except for the pressure are extrapolated which is determined by a 
Table 1 Supersonic and transonic freestream conditions

\begin{tabular}{ccccccc}
\hline $\mathrm{Ma}_{\infty}$ & $\mathrm{Re}, 1 / \mathrm{m}$ & $p_{\infty}, \mathrm{Pa}$ & $p_{0}, \mathrm{MPa}$ & $T_{\infty}, \mathrm{K}$ & $T_{0}, \mathrm{~K}$ & $u_{\infty}, \mathrm{m} / \mathrm{s}$ \\
\hline 6.0 & $10 \cdot 10^{6}$ & 633 & 1 & 52.4 & 430 & 871 \\
0.7 & $19 \cdot 10^{6}$ & $1.08 \cdot 10^{5}$ & 0.15 & 264 & 290 & 228 \\
\hline
\end{tabular}

characteristics approach. A sponge layer is applied in the base region to ensure acoustic pressure wave damping. At solid walls, an isothermal no-slip condition is imposed. Note that the simulations are compared to short time wind tunnel tests that lead to a negligible heating of the model. Periodic boundary conditions are used in the circumferential direction for the transonic case. At supersonic boundaries all variables are prescribed.

The Mach 6.0 freestream conditions correspond to a certain trajectory point of ESA's Ariane rocket at the Reynolds number of $10 \cdot 10^{6} 1 / \mathrm{m}$ and the total pressure of $1 \mathrm{MPa}$. Since the computational effort rises exponentially at increasing Reynolds number, the transonic conditions have to be adapted to Ariane's trajectory. The transonic uniform Reynolds number is set to $19 \cdot 10^{6}$, while the total pressure level is $0.5 \mathrm{MPa}$. A summary of all relevant freestream conditions is given in Table 1.

\section{RESULTS}

A schematic overview of the different flow phenomena that form the transonic and the supersonic wake flow is given in Fig. 5. At transonic flow, the backward facing step causes a separation of the boundary layer with a pronounced

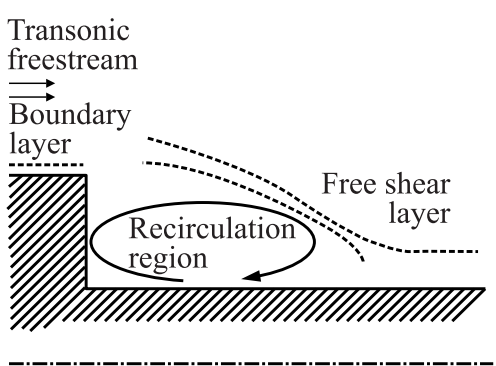

(a)

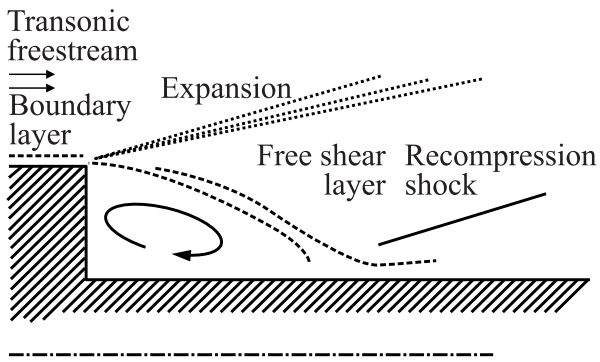

(b)

Figure 5 Schematic of flow characteristics in the transonic $(a)$ and supersonic $(b)$ wakes 
recirculation region. A free shear layer emanates from the sharp edge, curls up partially, and reattaches at the sting support. In the supersonic flow, however, an expansion at the sharp edge of the base shoulder occurs, and the shear layer is expected to build a smaller recirculation region downstream of the base. The subsequent wake is determined by a recompression shock, which deflects the flow to be parallel to the support and a pressure rise is observed. In the following, a more detailed description of the flow characteristics in the transonic and in the supersonic regime is presented.

\subsection{Transonic Wake Flow}

Since the configuration of the rocket model includes an aft sting representing the nozzle, the main body flow remains nearly undisturbed and can thus be

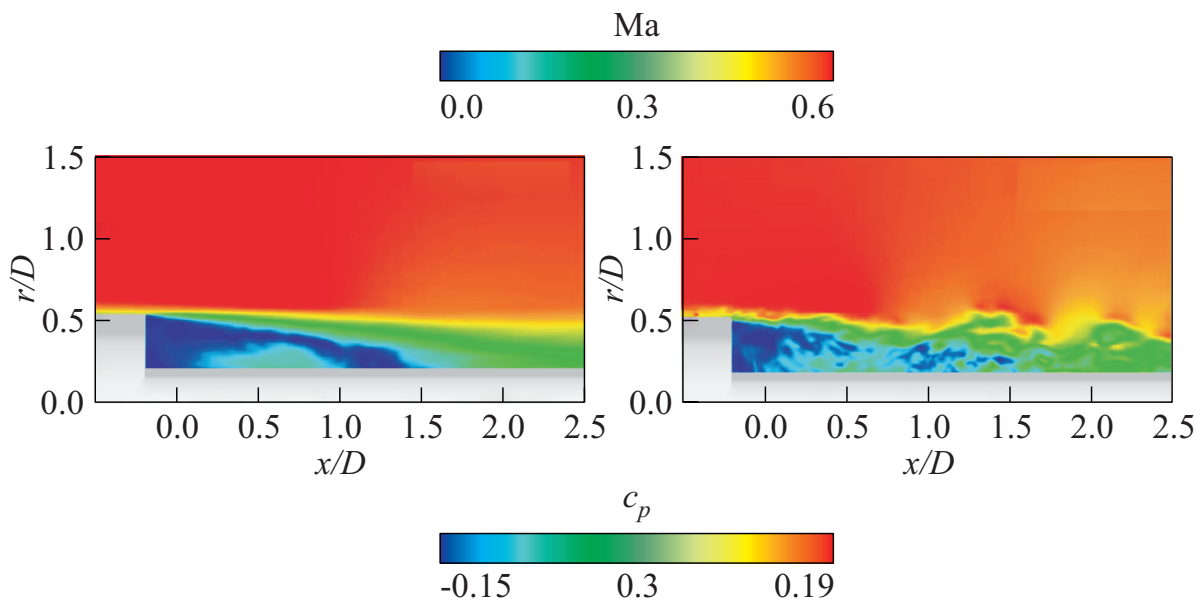

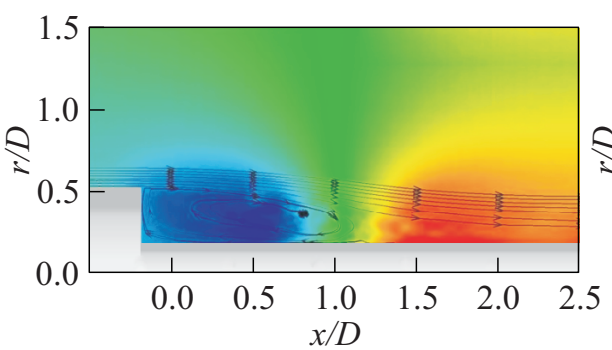

(a)

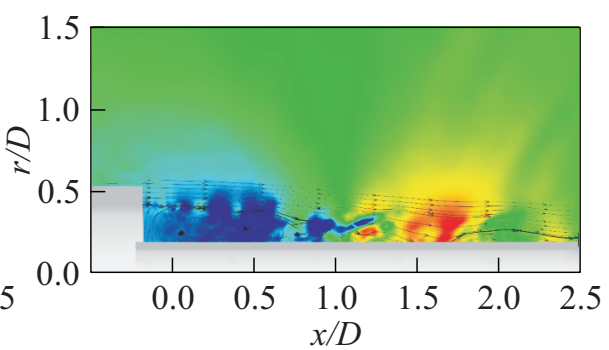

(b)

Figure 6 Mach number and pressure contours at transonic freestream conditions; mean $(a)$ and instantaneous $(b)$ distributions. 


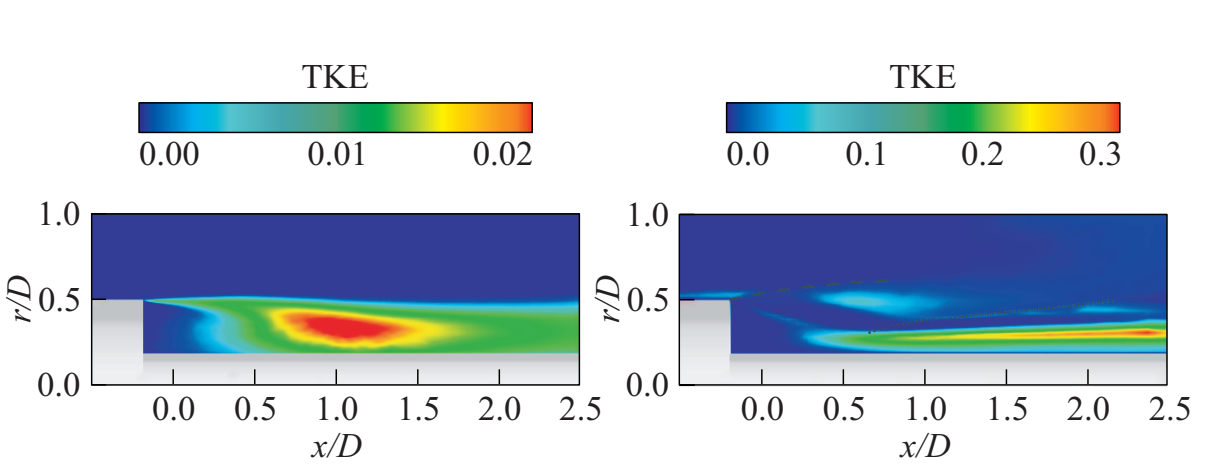

Figure 7 Turbulent kinetic energy distribution, transonic (a) and supersonic (b) flows.

considered axisymmetric since the angle of attack is $\alpha=0^{\circ}$. As was previously described, the transonic and supersonic wakes differ considerably.

Figure 6 shows the averaged and the instantaneous Mach number distribution in the near wake at transonic freestream conditions. The averaging is performed over a dimensionless time of about $100 \mathrm{t}$ corresponding to $25 \mathrm{~ms}$, with $\mathrm{t}$ being the characteristic time defined as the time a particle at freestream conditions needs to cover the reference distance, i. e., the main body diameter. The detached shear layer grows, while it slightly moves towards the centerline so that it impinges upon the support sting between $x / D=1.5$ and $x / D=2.5$. This encloses a recirculation zone of low Mach number at the base. The flow field is also evidenced by the averaged streamlines. The center of the recirculating vortex can be determined at about $x / D=0.7$. Moreover, Fig. 6 displays the averaged and the instantaneous distribution of the pressure coefficient $c_{p}=\left(p-p_{\infty}\right) /(1 / 2) \rho_{\infty} u_{\infty}^{2}$. The streamlines in the instantaneous pressure plot (see Fig. 6 ) indicate a motion of smaller eddy centers, which form the extensive recirculation bubble in the time-averaged illustration.

The turbulent characteristics, i.e., the turbulent kinetic energy (TKE), is shown in Fig. $7 a$. Most of the transonic turbulent structures appear in the wake, when the free shear layer evolves as vortex shedding in the region of the recirculation areas into a fully turbulent and chaotic motion with distinct flow direction towards the outflow boundary. The region of higher turbulent energy is enclosed by the outer boundary of the shear layer. Instantaneous distributions of the circumferential velocity component in the transversal plane at $x / D=0.25$ at two time states in Fig. $8 a$ illustrate complex nonaxisymmetric large-scale azimuthal flow structures even for the axisymmetric geometry. This flow structure is not evident in a time-averaged flow field.

To illustrate the dynamic effects of the recirculation zone on the rocket structure, distributions of the wall pressure coefficient in the radial and streamwise 

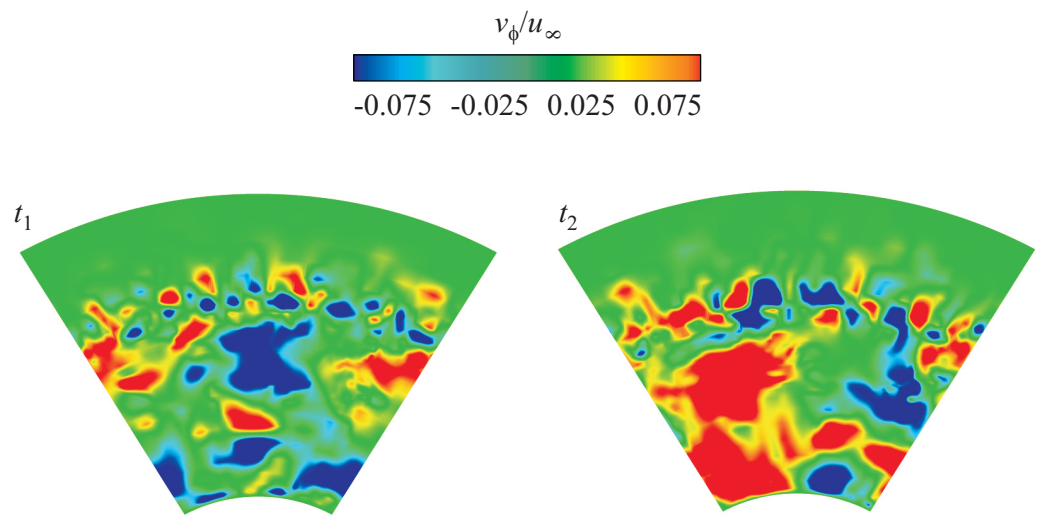

(a)
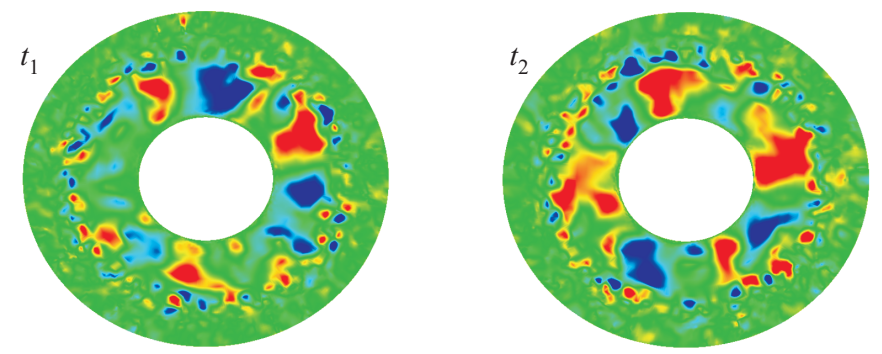

(b)

Figure 8 Circumferential velocity flow field of the transonic $(a)$ and supersonic $(b)$ configurations at two times $t_{1}$ and $t_{2}=4 D / u_{\infty}$.

directions are presented in Fig. 9. The base pressure coefficient possesses a nearly homogeneous radial distribution at a value of approximately $c_{p}=-0.148$. The radial root-mean-square ( $\mathrm{rms}$ ) distribution of the base pressure fluctuations exhibits a local maximum of $c_{p \text { rms }} \approx 9.8 \cdot 10^{-3}$ at $r / D=0.26$, then it decreases monotonically to $c_{p \mathrm{rms}} \approx 7 \cdot 10^{-3}$ at $x / D \approx 0.42$ and rises again at the outer base region. The streamwise evolution of the pressure coefficient on the surface of the support sting shows a small decrease of its value in the separated region up to $x / D \approx 0.6\left(c_{p} \approx-0.19\right)$ which is followed by a strong recompression indicating the beginning of the reattachment process further downstream. The maximum level is reached slightly downstream of the reattachment point at $x / D \approx 1.6$ $\left(c_{p} \approx 0.14\right)$. Further downstream, the pressure recovers at the freestream level. The streamwise rms distribution of the pressure fluctuations increases steadily 

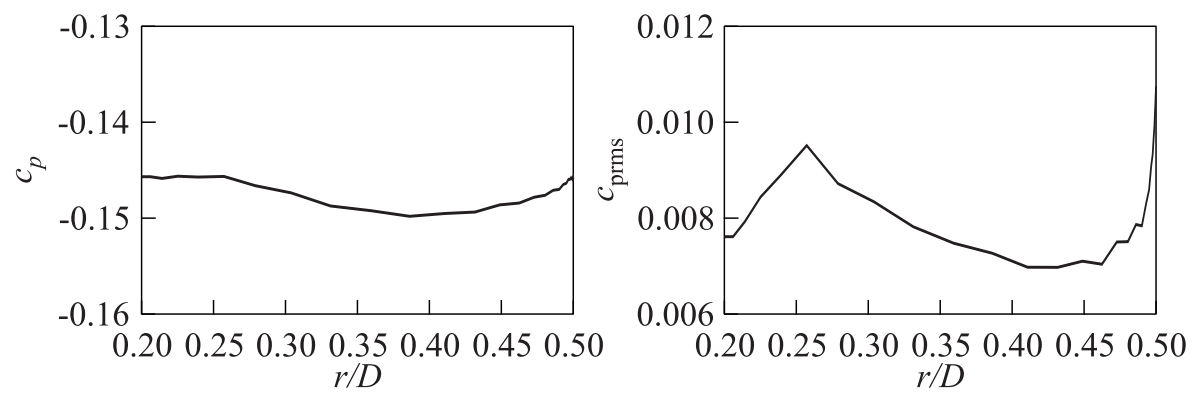

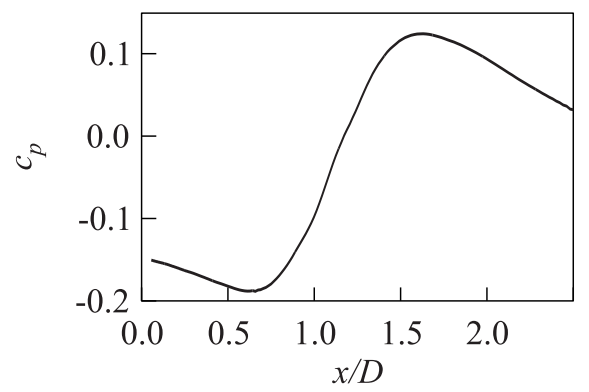

(a)

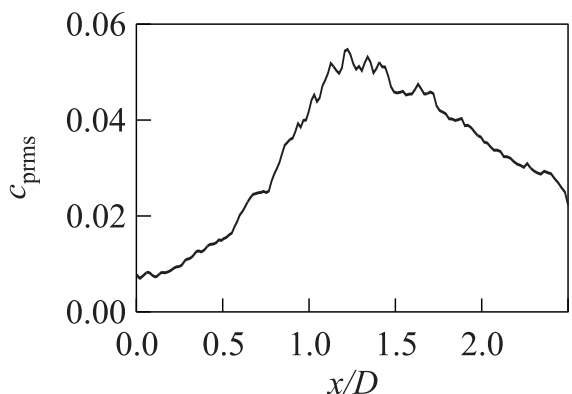

(b)

Figure 9 Radial (top) and streamwise (bottom) wall pressure coefficient distributions of the transonic configuration; mean $(a)$ and $\mathrm{rms}(b)$ distributions

up to its maximum of $c_{p \text { rms }} \approx 0.05$ just upstream of the reattachment position at $x / D \approx 1.4$.

The numerically computed wall pressure coefficient levels satisfactorily agree with experimentally measured values for similar configurations. For instance,
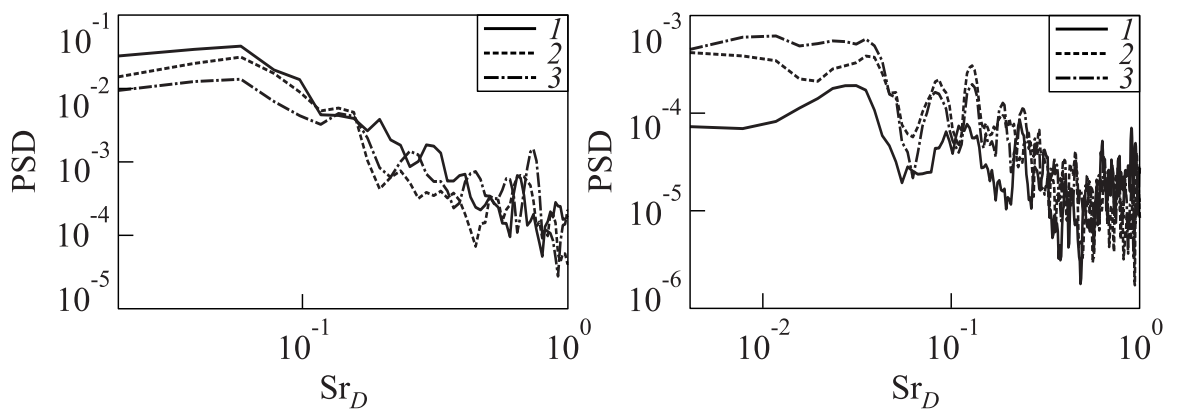

Figure 10 Base pressure spectra of the transonic $(a)$ and supersonic $(b)$ configurations: $1-r=0.24 D ; 2-0.35 D$; and $3-r=0.46 D$ 
Deprés et al. [14] determined the base pressure characteristics for an axisymmetric configuration with a cylindrical attachment $(L / D=1.2)$ at Ma $=0.85$ which scatter in the circumferential direction around $c_{p} \approx-0.13$ and $c_{p \text { rms }} \approx-0.011$. The streamwise evolution of the wall pressure coefficient and its rms value matches typical experimentally determined distributions and extrema found in the literature, e.g., Mabey [15], Deprés et al. [14].

To gain an insight into the dominant modes of the dynamic behavior, the power spectral density (PSD) distributions of the base pressure coefficient at three radial positions $r / D=(0.24 ; 0.35 ; 0.46)$ calculated by a DFT using a bin

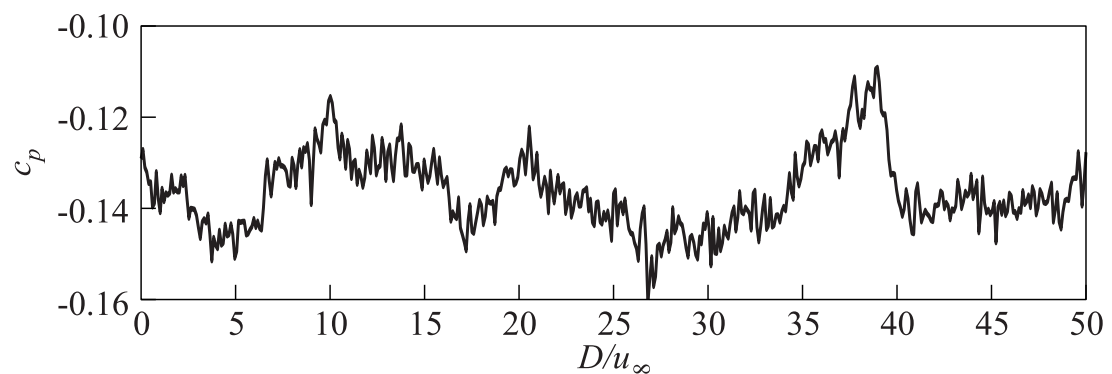

(a)

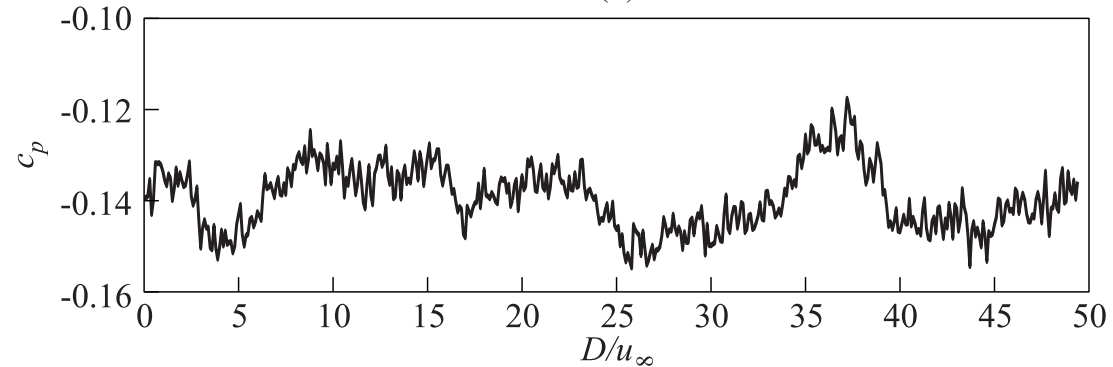

(b)

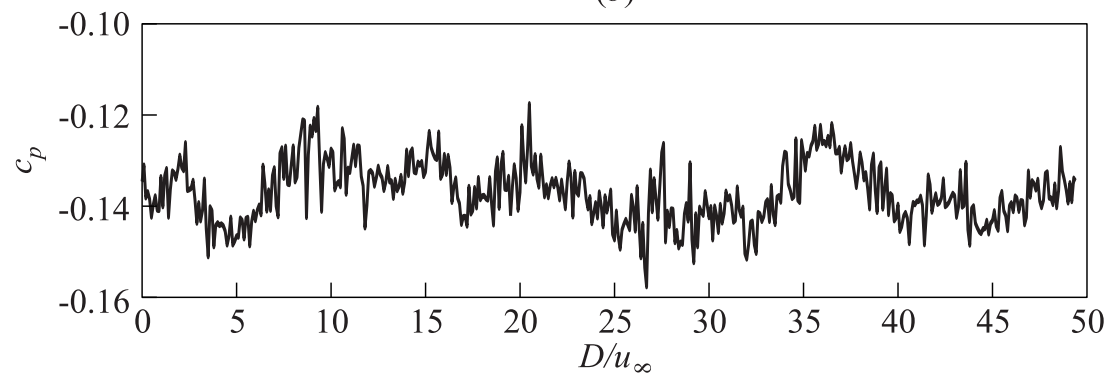

(c)

Figure 11 Base pressure fluctuations of the transonic configuration: $(a) r=0.24 D$; (b) $0.35 D$; and (c) $r=0.46 D$ 
smoothing over 3 bins are presented in Fig. $10 a$. The original time signal is shown in Fig. 11. The obtained spectra feature the first dominant mode at $\operatorname{Sr}_{D} \approx 0.058$ or $\operatorname{Sr}_{L r} \approx 0.08$ based on the length of the recirculation bubble which is equal to $L_{r} / D=1.4$. The same value determined by Deck and Thorigny [16] was shown to be a footprint of the vertical motion of the recirculation bubble (shear layer flapping). A second dominant peak at $\mathrm{Sr}_{D} \approx 0.2$ or $\mathrm{Sr}_{L r} \approx 0.28$ occurs for the wall pressure fluctuations at $r / D=0.25$. This is assumed to be related to the vortex shedding frequency which typically possesses values around $\operatorname{Sr}_{D} \approx 0.2$.

\subsection{Supersonic Wake Flow}

While the recirculation region for the transonic freestream conditions spans up to 1.4 diameter downstream of the base shoulder and possesses an eddy center at

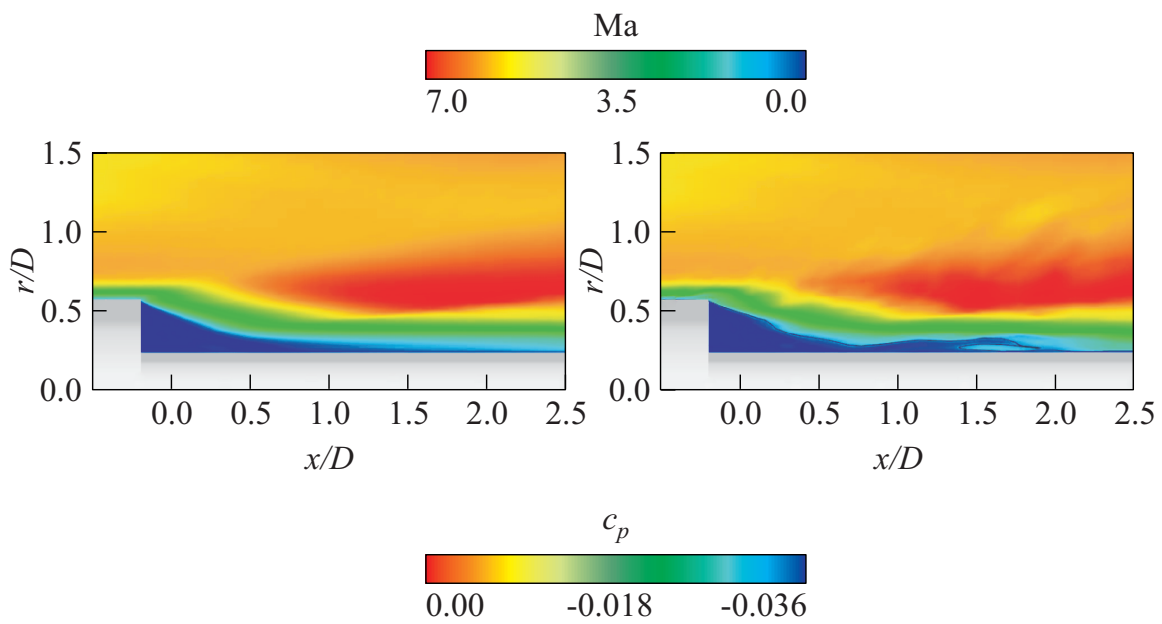

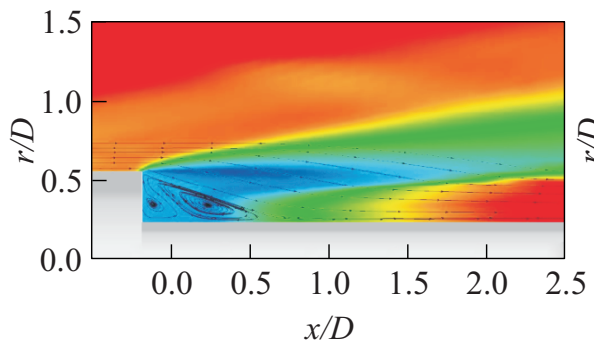

(a)

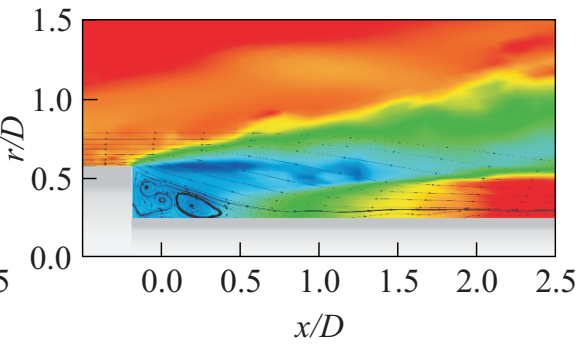

(b)

Figure 12 Mach number and pressure contours at supersonic freestream conditions; mean $(a)$ and instantaneous $(b)$ distributions. 
$0.7 D$, the streamwise extension of the recirculation area in the supersonic case is reduced by the factor of two. Moreover, the significantly higher velocities in the supersonic - compared to the transonic configuration - lead to a weaker growth rate of the shear layer, which seems to be constant over a wide range. It is evidenced in the Mach number distribution in Fig. 12. However, the low pressure recirculation zone at the base is considerably smaller. The shear layer is deflected towards the support right at the rocket's base shoulder. This is due to the expansion at the sharp edge, which bends the boundary layer towards the sting support. Further downstream, the deflected flow is redirected parallel to the support, which leads to a shock nearly parallel to the base expansion waves. Consequently, a region of low pressure establishes in the supersonic flow pattern, with its minimum right at the base plate.

A look at the instantaneous pressure coefficient distribution in Fig. 12 indicates that a major fraction of the turbulent energy of the incoming boundary layer is not transported in the shear layer as in the transonic case. It is characterized by the pattern of the expansion waves in an outward radial direction. This is confirmed by the turbulent kinetic energy plot in Fig. $7 b$, which reveals that the expansion fan leads to a decrease of the turbulent kinetic energy comprised in the boundary layer. Slight fluctuations being generated by the approaching
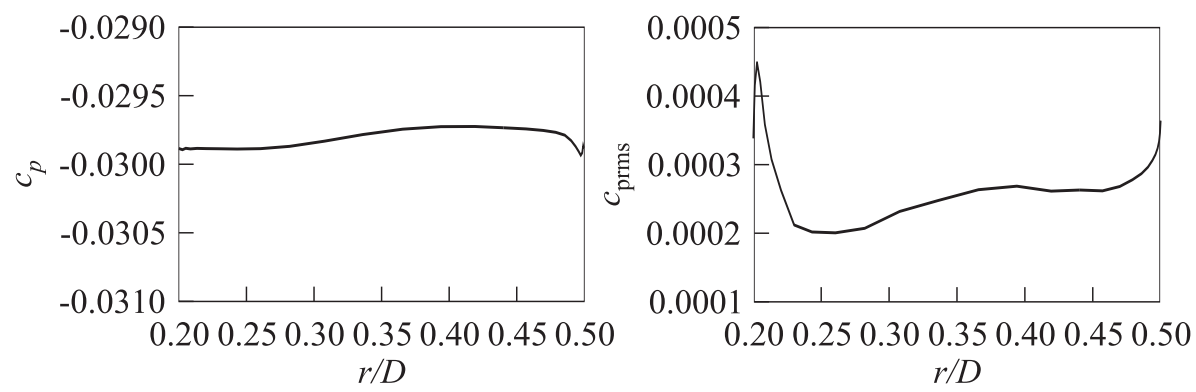

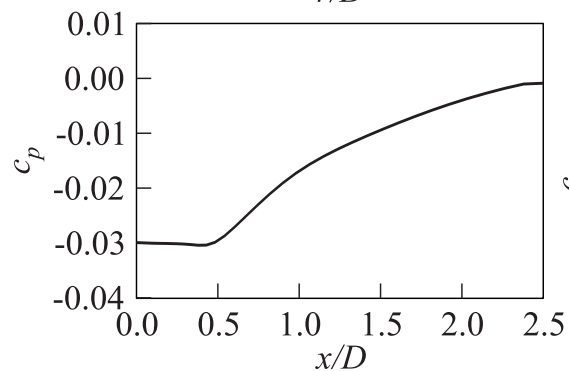

(a)

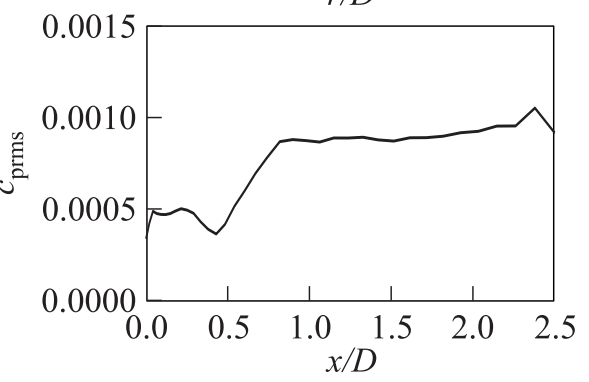

(b)

Figure 13 Radial (top) and streamwise (bottom) wall pressure coefficient distributions of the supersonic configuration; mean $(a)$ and rms $(b)$ distributions 


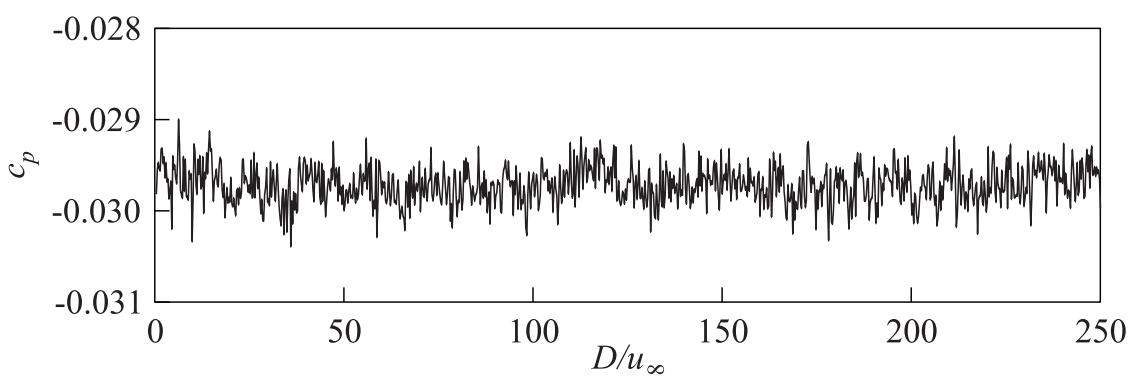

(a)

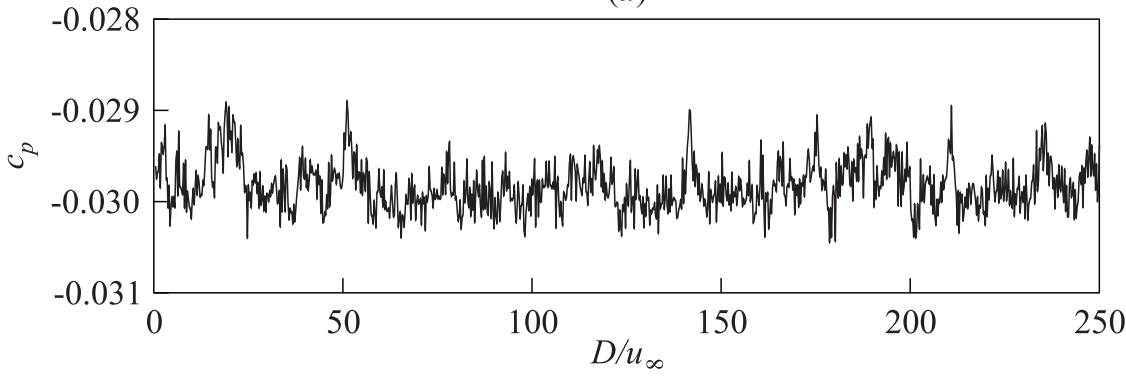

(b)

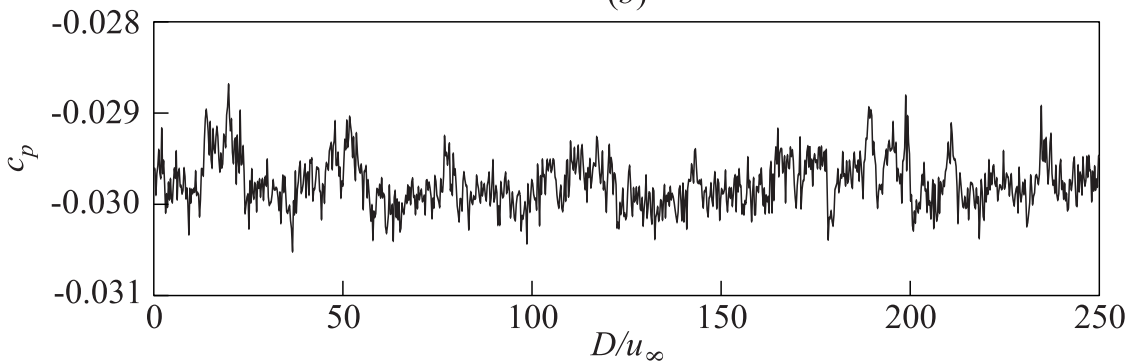

(c)

Figure 14 Base pressure fluctuations of the supersonic configuration: $(a) r=0.24 D$; (b) $0.35 D$; and $(c) r=0.46 D$

boundary layer are transported straight downstream and in the outward radial direction. Somewhat stronger fluctuations, however, are generated in the recirculation zones, which are transported downstream close to the support sting. The TKE is increased when the shock occurs (see Fig. 7). Compared to the transonic TKE, significantly higher levels appear. As in the transonic case, the instantaneous distribution of the circumferential velocity component in a transversal plane in Fig. $8 b$ demonstrates the presence of complex non-axisymmetric large scale azimuthal flow structures.

Similar to the transonic case, the radial and streamwise distributions of $c_{p \text { mean }}$ and $c_{p \text { rms }}$ on the wall are presented in Fig. 13 to reveal dynamic effects 
of the recirculation zone on the rocket structure at supersonic flow conditions. In the radial direction, the time averaged base pressure coefficient remains at the constant level of $c_{p} \approx-0.03$, whereas its rms value exhibits a distinct peak $\left(c_{p \text { rms }} \approx 0.00045\right)$ at the inner base corner at $x / D \approx 0.205$ which can be explained by high dynamic motion of a large scale toroidal secondary vortex described in [17]. In the streamwise direction, the mean pressure coefficient remains nearly constant up to $x / D \approx 0.5$ where the recompression shock occurs and subsequently, an increase in $c_{p}$ takes place. The excitation of the wall boundary layer by the recompression shock causes the wall pressure fluctuations to reach a higher constant level of $c_{p \text { rms }} \approx 0.001$ downstream of the shock position.

Figure $10 b$ presents the power spectral density (PSD) distributions of the base pressure coefficient at the radial positions $r / D=(0.24 ; 0.35 ; 0.46)$ calculated by a DFT using a bin smoothing over 6 bins. The original time signal is shown in Fig. 14. The supersonic spectra exhibit more distinct modes than the transonic configuration, i. e., $\mathrm{Sr}_{D}=(0.025 ; 0.08 ; 0.129 ; 0.192 ; 0.242)$ or $\mathrm{Sr}_{L r}=(0.018 ; 0.056 ; 0.09 ; 0.134 ; 0.169)$ based on the length of the recirculation bubble $L_{r} / D=0.7$. In [17], the first two modes are determined to be the footprints of the inner dynamics of the recirculation zone represented by two counter rotating and interacting toroidal vortices whose dimensions and core positions change in the time. Unsteady supersonic results show that the expansion remains mostly constant, while a coupled effect of the subsequent recompression shock and the recirculation region occurs, leading to a pulsating motion. Each unshaped flow circulation downstream of the base leads to a small shift in the position of the recompression shock and vice versa. The distinct mode of the recompression shock oscillations was determined experimentally and numerically in the range of $\operatorname{Sr}_{D} \approx 0.18$ [17]. The observed fourth corresponding mode in the base pressure spectra $\operatorname{Sr}_{D}=0.192$ at $r / D=0.46$ is assumed to be a footprint of this coupled effect.

\section{CONCLUDING REMARKS}

The base flow of a wind tunnel model of a generic rocket having a support sting mounted at its base was investigated in the trans- and the supersonic flow regime using a zonal RANS/LES method. While the RANS approach was used to predict the attached flow field upstream of the base shoulder, the LES was applied to calculate the unsteady flow in the wake of the main body and along the support sting.

The transonic and supersonic wakes differ considerably. While the recirculation region for the transonic freestream conditions extends up to $1.4 D$ downstream of the base shoulder and possesses an eddy center at $0.7 D$, the streamwise extension of the recirculation area in the supersonic case is reduced by a factor 
of two. This is due to the strong expansion at the sharp edge, which bends the boundary layer towards the sting support. It is also the shoulder expansion, which causes a static pressure in the recirculation region that is significantly lower than in the transonic case. Due to the acceleration of the flow, the expansion fan leads to a decrease of the turbulent kinetic energy comprised in the boundary layer. Unsteady supersonic results show that the expansion remains mostly constant, while a coupled effect of the subsequent recompression shock and the recirculation region can be observed. The turbulent kinetic energy again is increased through the recompression shock. Compared to the transonic TKE, significantly higher levels appear in the supersonic regime. Most of the transonic TKE appears in the wake, when the free shear layer evolves as vortex shedding in the region of the recirculation areas into a fully turbulent motion with distinct flow direction towards the outflow boundary. In the transonic and the supersonic regime, the recirculation region is dominated by motions of numerous small eddies, which only in a time-averaged illustration form distinct recirculation vortices.

\section{ACKNOWLEDGMENTS}

Financial support has been provided by the German Research Association (DFG - Deutsche Forschungsgemeinschaft) in the framework of the Transregional Collaborative Research Center TRR40. Computational resources have been provided by the Stuttgart High-Performance Computing Center (HLRS).

\section{REFERENCES}

1. Rollstin, L. 1987. Measurement of inflight base-pressure on an artillery-fired projectile. AIAA Paper No. 287-2427.

2. Liou, M. S., and C. J. Steffen. 1993. A new flux splitting scheme. J. Comput. Phys. 107:23-39.

3. van Leer, B. 1979. Towards the ultimate conservative difference scheme. V. A second-order sequel to Godunov's method. J. Comput. Phys. 32:101-36.

4. Fureby, C., and F.F. Grinstein. 2002. Large eddy simulations of high-Reynoldsnumber free and wall-bounded flows. J. Comput. Phys. 181:68-97.

5. Boris, J. P., F.F. Grinstein, E. S. Oran, and R. L. Kolbe. 1992. New insights into large eddy simulation. Fluid Dyn. Res. 10(4-6):199-228.

6. Spalart, P. R., and S. R. Allmaras. 1992. A one-equation turbulence model for aerodynamic flows. AIAA Paper No. 92-0439.

7. Spille, A., and H.-J. Kaltenbach. 2001. Generation of turbulent inflow data with a prescribed shear-stress profile. 3rd AFSOR Conference on DNS and LES. 
8. König, D., M. Meinke, and W. Schröder. 2010. Embedded LES/RANS boundary in zonal simulations. J. Turbulence 11:1-25.

9. Zhang, Q., W. Schröder, and M. Meinke. 2010. A zonal RANS-LES method to determine the flow over a high-lift configuration. Computers Fluids 39(7):1241-53.

10. Keating, A., G. de Prisco, and U. Piomelli. 2006. Interface conditions for hybrid RANS/LES calculation. Int. J. Heat Fluid Flow 27:777-88.

11. Jarrin, N., N. Benhamadouche, S. Laurence, and D. Prosser. 2006. A syntheticeddy-method for generating inflow conditions for large-eddy simulations. J. Heat Fluid Flow 27:585-93.

12. Meiß, J.-H., M. Meinke, and W. Schröder. 2012 (in press). Large-eddy simulation of nozzle-base-flow interaction. J. Spacecraft Rockets.

13. Roidl, B., M. Meinke, and W. Schröder. 2012. A zonal RANS-LES method for compressible flows. Computers Fluids 67:1-15.

14. Deprés, D., P. Reijasse, and J.P. Dussauge. 2004. Analysis of unsteadiness in afterbody transonic flows. AIAA J. 42(12):2541.

15. Mabey, D. G. 1972. Analysis and correlation of data on pressure fluctuations in separated flows. J. Aircraft 9(9):642-45.

16. Deck, S., and P. Thorigny. 2007. Unsteadiness of an axisymmetric separating-reattaching flow: Numerical investigation. Phys. Fluids 19:065103, doi:10.1063/1.2734996.

17. Saile, D., A. Gülhan, A. Henckels, C. Glatzer, V. Statnikov, and M. Meinke. 2013. Investigations on the turbulent wake of a generic space launcher geometry in the hypersonic flow regime. In: Progress in flight physics. Eds. Ph. Reijasse, D. Knight, M. Ivanov, and I. Lipatov. Advances in aerospace sciences EUCASS book ser. $5: 209-34$. 